Hamma, B. (2019a), in Dir. Cécile Avezard-Roger, Céline Corteel, Jan Goes, Belinda Lavieu-Gwozdz: La phrase, carrefour linguistique et didactique. Artois Presses Université, p. 281-301.

\title{
Pour une didactique de la diamésie : revers du recours à la phrase forgée dans l'enseignement (cas de la litote et du passif)
}

\author{
Badreddine HAMMA \\ Université d'Orléans - LLL, UMR 7270
}

Le but de cette étude est de montrer en quoi le recours à des phrases forgées dans l'enseignement de la grammaire et du français de façon générale peut entraîner de fâcheuses conséquences sur la qualité de l'apprentissage et peut, de ce fait, être une source non négligeable d'échec pour les apprenants. Notre argumentation s'appuiera sur deux tours très récurrents dans les cours de langue : le passif et la litote. Nous défendrons ainsi la nécessité d'abandonner une exemplification figée, rigide et forgée ou se cantonnant à un écrit hors contexte, pour une approche plus écologique des exemples, qui ne perde pas de vue les dimensions énonciative, fonctionnelle et variationnelle, ce qui implique une nécessaire extension aux données orales. Nos propositions sont adossées, ici, aux corpus ESLO (les Enquêtes SocioLinguistiques à Orléans).

Notre propos sera ainsi de considérer certaines situations d'échec auxquelles peut être confronté un apprenant en classe de langue, en vue de déterminer avec précision leurs origines et de proposer des solutions visant à optimiser les méthodes d'enseignement existantes: l'échec dont il est question, ici, peut relever aussi bien d'un ratage communicatif banal que d'un ratage au niveau des objectifs pédagogiques institutionnels, aboutissant à un échec scolaire. Nous montrerons en particulier en quoi l'intégration d'une compétence « diamésique » $(\mathbf{5})$ dans l'enseignement des langues pourra s'avérer salutaire pour vaincre ce type de difficultés à travers l'exemple du français.

\section{1-Malaise communicatif et malaise scolaire : constat de l'échec}

Les situations d'échec dont il est question ici se voient chez l'apprenant à la fois dans l'acte d'encodage, à travers, par exemple, une intention de signification inaboutie, ou une tentative manquée de s'exprimer, etc., et, dans l'acte de décodage, à travers une méprise, ou une incapacité de cerner le sens de ce que l'on peut lui adresser dans un échange commun. Nous pouvons penser à ces grands moments de solitude que peut connaître un étranger, quand il est le seul, par exemple, à ne pas rire d'une blague, qui fait pourtant rire tout le monde autour de lui. Ces petits échecs du quotidien sont loin d'être anodins, vu qu'ils sont susceptibles, à long terme, d'entraîner de plus gros échecs ; ils deviennent des facteurs de dissuasion et de démotivation pour, par exemple, l'apprenant de FLE, qui prend alors conscience de son impuissance devant tout ce qui reste à apprendre et tout ce qu'il ignore ou méconnaît des «subtilités » de la langue cible. De telles considérations sont à mettre 
aussi en relation, sur le plan affectif, avec la notion de "face » de Goffman (1973), qui s'invite dans les interactions sociales et met en jeu l'estime de soi : le sujet parlant aspire constamment à communiquer une bonne image de soi-même et à exercer une certaine autorité à travers le langage ( $c f$. Bourdieu, 1982). Cela n'épargne pas non plus le natif, qui pourrait avoir l'impression que sa propre langue lui échappe ; le FLM ou le français langue première (FLP) à l'école devient une vraie " langue étrangère » pour les francophones euxmêmes : il s'agit souvent d'une variété de français qui rompt avec la langue qu'ils utilisent. En effet, un sujet natif peut très bien mélanger les genres de discours, faire de fâcheux amalgames d'usages et confondre les étiquettes que l'on associe à des faits langagiers ordinaires de sa langue maternelle. Et cela se voit surtout à l'écrit. C'est le cas de l'élève qui rend une copie blanche parce qu'il n'a pas su conjuguer un verbe à l'imparfait ou à l'impératif, ou qu'il n'a pas pu remplir un texte à trous sur le complément circonstanciel adéquat, pourtant il est censé maîtriser parfaitement ces aspects grâce à sa compétence linguistique de natif. De fait, ici, c'est surtout l'absence de liens avec l'usage effectif de ces faits de langue, si communs, qui lui pose problème.

Ces échecs sont visibles aussi quand l'apprenant étranger, au bout du ompte, "parle comme un livre » et quand l'élève natif écrit parfois comme il parle dans une dissertation, compte tenu de sa méconnaissance des frontières entre l'oral et l'écrit. Et, au-delà de ces manifestations locales au quotidien, ces impérities se voient dans des phénomènes plus larges, comme le « dégagement » (Cordié, 1993), l'échec scolaire et le décrochage précoce. Ces difficultés peuvent, certes, avoir différentes sources, qui vont des facteurs économiques et sociologiques aux facteurs politiques, en passant par les facteurs psychologiques et, dans certains cas, physiologiques ( $c f$. Ravon, 2000), il existe aussi, comme annoncé supra, une grande part de responsabilité qui est liée, selon nous, à la politique d'enseignement et à la nature des contenus dispensés à l'école. C'est, en tout cas, la rupture entre l'exercice scolaire et la pratique de la langue en dehors de la salle de classe, qui nous intéresse ici. Plus précisément, nous nous focaliserons sur les revers de l'absence des « compétences diamésiques » dans l'enseignement du français - résultat du recours récurrent aux phrases forgées et artificielles dans l'exemplification scolaire.

\section{2-Place de l'oral dans l'enseignement en théorie et en pratique}

Dans les textes officiels et dans les référentiels de formation, l'oral, en général, semble occuper une place très importante. Ainsi, personne ne conteste son intérêt en contextes de FLE, par exemple ; d'ailleurs, il en va de l'efficacité de la démarche et de la qualité des résultats obtenus. En effet, le principal problème des apprenants étrangers n'est pas tellement celui d'écrire correctement le français que de s'exprimer d'une manière naturelle, claire et économique. Souvent le niveau avancé des apprenants en FLE ne les empêche pas de tomber dans des situations désagréables à l'oral et de risquer leur « face positive » (voir Goffman, 1973). C'est d'ailleurs dans cet esprit que les compétences orales sont préconisées par le CECRL (Cadre Européen Commun de Référence pour les Langues) dans l'apprentissage des langues étrangères: la certification doit d'ailleurs rendre compte du 
niveau de maîtrise de l'oral à la fois dans les compétences de compréhension, de production et, plus avant, dans celles d'interaction.

En contextes de FLM, si l'écrit est privilégié, l'oral y occupe aussi une place qui n'est pas moindre dès le primaire, comme en témoigne ce qui est stipulé dans les programmes officiels en France ( $c f$. BO 2015, p. 17-18) :

Développer la maitrise de l'oral suppose d'accepter essais et erreurs dans le cadre d'une approche organisée qui permette d'apprendre à produire des discours variés, adaptés et compréhensibles permettant ainsi à chacun de conquérir un langage plus élaboré.

Les grilles des compétences des paliers 1, 2 et 3 du SCCC (2016, p.3) (Socle Commun Des Connaissances, Des Compétences et de Culture) vont aussi dans ce sens quant à la place de l'oral à l'école.

Compte tenu de ces positions, on pourrait s'attendre à ce que l'oral ait une place conséquente, qu'il soit suffisamment représenté dans les pratiques scolaires, a fortiori quand il est question d'expliquer le fonctionnement de la langue enseignée, qu'elle relève d'une langue maternelle ou d'une langue seconde ou étrangère. Cette « évidence », comme nous allons le voir, ne semble exister malheureusement que sur le papier.

\section{3- Place de l'oral dans le discours didactique}

Le terme « oral » dans les pratiques pédagogiques est généralement très présent et recouvre plusieurs réalités; il peut renvoyer aussi bien aux pratiques de prononciation, qu'aux activités d'oralisation et à l'expression de soi, qui englobent la lecture à haute voix de textes écrits, ou de dialogues, la récitation, ou indirectement à travers différents types d'activités de classe (prise de la parole, jeux de rôles, mises en situation, etc.). La seule réalité qui semble manquer à l'appel est le recours à des données orales authentiques dans la conception des supports pédagogiques au profit des données écrites, ainsi que les outils utilisés pour les décrire et les interpréter. Le prestige de l'écrit à l'école se manifeste, ainsi, à travers un recours quasi exclusif dans l'enseignement de la grammaire à des phrases forgées ou écrites, prises dans des textes d'auteurs et, dans les meilleurs des cas, dans la presse, ou encore, depuis la méthodologie audio-orale (MAO) et, à sa suite, la méthodologie structuro-globale audiovisuelle (SGAV), à des extraits radiophoniques ou télévisés et, très souvent, à des dialogues fabriqués. Mais, la radio, la télévision, avec les séries, les films, les publicités, les émissions socioculturelles, les jeux, etc., tout comme pour les autres supports écrits, bénéficient tous, à des degrés variés, d'une certaine préparation, d'une certaine élaboration préméditée et cadrée qui sont étrangères à ce que l'on peut observer dans les interactions orales, où la construction du sens se fait de manière hic et nunc. Cette préparation est de nature à annihiler et à fausser la « teneur » discursive, l'investissement élocutif et les interactions qui caractérisent l'énonciation dans les 
exemples scolaires (Hamma 2017 à par.). Somme toute, la langue parlée en tant que produit sociolinguistique spontané et comme lieu de variations, semble absente de l'exemplification scolaire : le côté imprévisible, incontrôlé et relâché du discours oral est considéré comme une maladresse de style qu'on cherche à éviter dans les supports pédagogiques, sans doute, pour ne pas donner le «mauvais exemple » aux apprenants, ce qui est, selon nous, une grosse erreur, à plus forte raison en contextes de FLE: un apprenant, à qui on ne cesse de répéter, par exemple, qu'il ne faut jamais oublier le ne de négation et qu'il faut toujours bien prononcer le schwa dans des mots comme seconde, acheter, petit, le, me... sera déstabilisé et aura l'impression d'avoir appris des choses inutiles, fausses ou insuffisantes, quand il se trouve immergé dans une « vraie » situation de communication avec des natifs, à l'aéroport, au guichet de la SNCF ou dans les rues de France et où ces formes ont tendance à disparaître. Les structures normées et rigides qu'il a apprises volent en éclats ! Il en va de même des contextes de FLM, où l'usage de l'oral ne devrait pas être une simple option : en effet, l'enfant, comme le rappelle, à juste titre, Tisset (2010, p. 57), n'accède pas à sa langue maternelle à travers la «phrase », mais à travers les interactions et les énoncés oraux, qui ne suivent pas forcément l'ordre canonique «SujetVerbe-Complément » rencontré dans les manuels scolaires, ce qui est susceptible de poser de grandes difficultés à l'élève en classe, vu qu'il aura, à la fois, à gérer les connaissances métalinguistiques expliquées et à s'adapter à un discours très peu naturel, à un mode d'expression artificiel et, en tout cas, non représentatif de sa langue.

Il devient ainsi difficile de défendre l'idée que le FLM (ou le FLP) tel que pratiqué dans nos écoles soit une « langue vivante », comme le fait remarquer Guerin (2014, p.104), à la suite de Vigner (2003). La place de l'oral en classe de français pose problème à deux égards : en amont, il faudrait que les concepteurs d'outils pédagogiques et les décideurs des programmes ministériels prennent réellement conscience du rôle joué par l'oralité. Et, en aval, il faudrait insister sur l'intérêt d'y recourir en classe auprès des enseignants, ce qui ramène à la question de la formation des formateurs. Le but serait de supplanter les phrases forgées par des énoncés à vocation authentiques pris, par exemple, dans les bases de données orales élaborées par des linguistes et des sociolinguistes, en veillant à leur représentativité et à leur variabilité.

\section{4- Méthodes : objectivation du constat d'échec}

Pour établir le constat d'échec dont nous parlons ici et légitimer nos propositions, nous avons essayé de transformer un constat subjectif, issu de 20 ans d'expérience dans l'enseignement et de retours d'expériences ${ }^{1}$, en une donnée mesurable et objective, et ce, à travers des enquêtes que nous avons supervisées en collège, lycée et université sous forme

\footnotetext{
${ }^{1}$ Sous forme de stages, de mémoires, etc. d'étudiants de master « Linguistique \& didactique » à l'Université d'Orléans.
} 
de questionnaires auprès de différents profils ${ }^{2}$. Le but de ces enquêtes était d'évaluer ce que les élèves retenaient des savoirs scolaires pendant ou après la formation suivie, ce qui permet a priori d'apprécier le rendement des méthodes d'acquisition employées, partant du postulat qu'un savoir non renforcé par l'application est exposé à l'oubli et à la confusion.

Pour pouvoir apprécier objectivement la qualité de l'acquisition des savoirs scolaires retenus et déterminer si l'apprentissage aura été réussi et concluant ou non, nous avons mis en place une stratégie d'évaluation qui entend rendre compte d'une manière plus fiable du degré de maîtrise d'un savoir donné. Notre méthode consiste à poser quatre conditions bien précises permettant de préjuger scientifiquement des compétences évaluées : soit $\boldsymbol{x}$ un savoir donné ayant fait l'objet d'un apprentissage par un sujet donné en classe de français. $\mathrm{Du}$ point de vue cognitif, l'acquisition et l'assimilation de ce savoir suit un certain cheminement qui garantit la fiabilité et la durabilité de l'apprentissage. On admettra ainsi que l'appropriation d'un fait linguistique $\boldsymbol{x}$ se reconnaît aux indices suivants ; il faudrait :

a) être capable de nommer $\boldsymbol{x}$ ou de l'intégrer dans une catégorie métalinguistique (ce qui pourrait poser le problème de la terminologie utilisée) ;

b) être capable de définir $\boldsymbol{x}$, y compris en utilisant ses propres mots (ce qui permettrait d'éviter le problème posé par le point (a)) ;

c) être capable de donner des exemples, nouveaux et différents, illustrant $\boldsymbol{x}$.

d) être capable de reconnaître $\boldsymbol{x}$ dans son milieu naturel : le discours.

Nous avons essayé ainsi de tenir compte de ces conditions pour l'élaboration de notre questionnaire. Il fallait définir les deux faits de langue choisis $(\boldsymbol{x})$, dire quel en est l'intérêt dans l'usage, en donner des exemples et enfin procéder à une identification de $\boldsymbol{x}$ à partir d'une sélection d'exemples se présentant sous forme de QCM.

Pour éviter d'avoir des réponses approximatives et fortuites, nous avons prévu pour la litote des exemples qui contiennent tous une litote avec des variations diaphasiques et diamésiques. Parmi les exemples cités, nous avons mis le fameux exemple qui cristallise dans la mémoire discursive des formateurs (et partant, chez beaucoup d'apprenants) ce qu'est une litote, à savoir la phrase de Chimène adressée à Don Rodrigue dans Le Cid de Corneille ( $\mathrm{Va}$, je ne te hais point!), en réponse à un soupçon que Rodrigue a eu quant aux sentiments de Chimène pour lui, surtout qu'il venait de tuer son père - contexte souvent ignoré par les usagers.

\section{a) Qu'est-ce qu'une litote?}

\footnotetext{
${ }^{2}$ Il s'agit de 126 élèves de $3^{\text {ème }}$ du collège Rosa Parks à Châteauroux, de seconde du Lycée Jean-Zay à Orléans) et de 152 étudiants de licence et de master inscrits au département des sciences du langage à Orléans. J'en profite pour remercier Jocelyne Pousset et Florence Piard pour leur aimable collaboration dans la réalisation de ces enquêtes.
} 
b) À quoi sert-elle?

c) Donnez un exemple de litote.

d) Y a-t-il une litote dans les exemples suivants?

1. Va, je ne te hais point!

2. Il est pas mal, ce film!

3. Max n'est pas un gamin pour croire à cette histoire!

4. Son charme ne me laisse pas indifférent.

Et pour le passif, nous avons repris également des exemples qui renferment, tous, divers emplois passifs, à l'exception de (1) et (3), où l'on a glissé une donnée subliminale rappelant la célèbre phrase, usée, donnée en (2) : La souris est mangée par le chat (cf. Hamma, 2017, à par.). Cette phrase pourrait leurrer l'élève inattentif en cas d'incertitude, surtout avec la présence de souris et de chat ou aussi la ressemblance formelle avec le passif, avec être, un participe passé et un complément en par. Cela constituera un excellent catalyseur pour la tâche demandée :

a) Qu'est-ce qu'une phrase au passif?

b) À quoi sert-elle?

c) Donnez un exemple de phrase passive.

d) Les exemples suivants sont-ils au passif?

1. La souris est sortie par la fenêtre.

2. La souris est mangée par le chat.

3. Le chat est fatigué.

4. Max s'est fait virer.

5. Le café ne se boit que chaud.

6. L'exploitation de l'homme par l'homme.

7. Cette écriture est illisible.

8. Le français employé par notre prof est très difficile.

Notons que pour la question (d), les exemples au passif, ne renvoient pas forcément à des cas canoniques et sont diaphasiquement différents : on retrouve un factitif en (4), un pronominal en (5), une nominalisation passive en (6), un passif lexicalisé, formé du suffixe -ble en (7) et, enfin, un passif sans l'auxiliaire être, en (8). Nous avons délibérément placé la tâche $(\mathrm{d})$ de reconnaissance à la fin du questionnaire pour permettre justement aux enquêtés de répondre après un certain effort de réflexion sur les fonctions respectives de la litote et du passif. Les seuls enjeux pour les étudiants se résumaient a priori à la question de défi à relever en essayant de répondre correctement aux questions. Pour les étudiants interrogés, comme nous avons procédé à une enquête orale en amphi et en salles de classe, la tâche d'exemplification (c) n'a pas pu être faite d'une manière systématique. En revanche, une justification des réponses fournies était possible à travers des compléments d'information et des demandes de reformulation. 
Nous avons procédé, par la suite, à une évaluation globale des 268 réponses recueillies, visant à déterminer globalement le niveau des enquêtés et de jauger indirectement la qualité des méthodes d'enseignement employées.

Les résultats obtenus s'avèrent assez inquiétants et confirment le constat subjectif de départ. Cela traduit une grande méconnaissance, voire une ignorance surprenante des faits étudiés et de leur utilité. La première chose qui frappe est la quantité de blancs laissés, qui peuvent certes s'expliquer par l'absence d'enjeux pour les enquêtés, mais qui reflètent surtout un vide ou un certain flou au niveau des apprentissages. Les non-réponses laissées s'élèvent pour la question (a) à $54,57 \%$ pour la définition du passif et à $67,41 \%$ pour la litote et pour la question (b), concernant leur intérêt respectif, cela atteint $74,9 \%$, pour le passif et 55,64\% pour la litote. Ce qui parait d'ailleurs symptomatique du flottement des savoirs appris est le fait qu'une grande partie des réponses données comme définition de $\boldsymbol{x}$ s'interprétait, en réalité, comme une glose de son utilité et parfois c'était l'inverse qui se produisait ; c'est ainsi que la litote est souvent définie en (a) par la glose « Dire moins pour suggérer plus » et l'intérêt évoqué du passif n'est autre qu'un élément de définition parmi les éléments appris : «C'est un temps de conjugaison » (9 réponses), «C'est quand le sujet subit l'action » (28 réponses) ou aussi «c'est quand on inverse l'ordre dans la phrase » (20 réponses). Du reste, les réponses fournies font preuve de nombreux amalgames : la litote est souvent confondue avec l'euphémisme, l'antiphrase, la périphrase ou l'antithèse. Cela signifie que l'assimilation de tous ces autres procédés pose autant problème et probablement pour les mêmes raisons ; l'exemplification utilisée ne permet pas d'y accéder. De plus, certaines réponses fournies reflétaient un contresens certain; ainsi, la litote est parfois vue comme une figure d'insistance (4 réponses) et le passif est défini comme une figure de style ou qui sert à exprimer « le contraire de ce que dit la phrase active » et deux réponses disant que le passif «ne sert à rien»! Mais parmi les réponses les plus significatives relevées ( 8 réponses), on a «Je ne sais plus »/«je ne me rappelle plus »: l'information en question aura été mémorisée pour un certain temps et, à défaut d'un renforcement par le réemploi, aura fini par être oubliée. Notons aussi que les étudiants interrogés oralement sur la litote et ayant fourni une réponse acceptable étaient globalement incapables de reformuler la définition en utilisant leurs propres mots.

Pour la tâche de reconnaissance et d'exemplification, les non-réponses étaient aussi très élevées. On recense ainsi seulement 29,96\% réponses pour le passif et 32,1\% pour la litote. Ces pourcentages ne tiennent d'ailleurs pas compte des mauvaises réponses et correspondent en réalité à des réponses partielles : pour le passif, nous avons considéré que l'exercice était concluant à partir de 4 bonnes réponses sur 8 et pour la litote, il suffisait d'en reconnaître 2 sur les 4 présentes. Notons que 17 sujets sont tombés dans le piège commenté ci-dessus, en reconnaissant (1) et (3) comme des passifs. Quant aux exemples personnels, les abstinences étaient encore plus élevées : seulement $26,75 \%$ ont répondu pour le passif et $17,12 \%$ pour la litote et là encore, les exemples donnés sont généralement 
calqués sur les exemples fournis dans le questionnaire ou reconnus comme illustrant l'une de ces deux tournures.

Ces difficultés sont, ainsi, le meilleur porte-parole de l'inanité et de l'inefficacité de certaines stratégies pédagogiques utilisées dans nos écoles. Et mis à part quelques cas d'incompréhension liés à l'absence d'enjeux, ou à la manière dont les questionnaires ont été présentés, ces mauvais résultats, selon nous, s'expliquent avant tout par l'absence d'une exemplification appropriée dans les pratiques scolaires; le choix de phrases forgées, non écologiques (phrases monologales exagérément simplifiées et sans ancrage dans l'usage) font que les formes apprises restent seulement dans la mémoire de travail des apprenants, qui les apprennent par cœur juste pour passer un examen par exemple, ce qui ne fait que renforcer la rupture entre les savoirs enseignés et leur utilité effective. De fait, la meilleure solution sera, sans doute, d'intégrer la diamésie dans les compétences communicatives en classe de langue.

\section{Pour une intégration de la compétence diamésique en classe de français}

\subsection{Qu'est-ce que la « compétence diamésique » ?}

La notion de « diamésie » renvoie globalement à la variation de chenal dans une langue donnée qui concerne, en l'occurrence, l'oral et l'écrit selon Gadet (1996, p. 25), succédant à Mioni (1983, cité par Wüest, 2009), qui assimile cette distinction aux variations «diaphasiques ». La séparation entre ces deux modes de communication est, certes, "fragile », comme le souligne Gadet (ibid.), qui y voit une sorte de « continuum », vu que les frontières entre l'écrit et l'oral ne sont pas franches; l'auteur rappelle, toutefois, la nécessité de les distinguer sur le plan de la description. Quant à la « compétence diamésique », ce serait, parallèlement à la « littératie », cette nécessaire aptitude qu'un sujet devrait acquérir et développer dans les pratiques de la communication et qui se définit comme la disposition à reconnaître et à utiliser l'aspect formel ou informel dans un discours, à bon escient : le locuteur doit être capable de moduler lucidement son discours, en fonction du contexte de communication qui se présente, ce qui implique une maîtrise des outils d'analyse et d'interprétation de chacun des deux types d'actualisation langagière. Et c'est justement cette dimension «diamésique» qui semble globalement faire défaut dans l'enseignement du français de manière générale et que nous voulons introduire et affermir dans les pratiques de classe. C'est la raison pour laquelle nous considérons que la part de responsabilité du système éducatif, de l'école et de la qualité des programmes y joue un rôle crucial. Il n'est plus idéaliste, ni alarmiste, par conséquent, de remettre en cause l'intérêt de certains savoirs scolaires tels qu'enseignés dans nos écoles, ou aussi dans certaines méthodes de FLE (Le Bellec \& Hamma, 2014), s'ils ne tiennent pas compte de la réalité de leur usage dans le discours : ils seraient tout simplement vains et déroutants pour les apprenants. Il ne s'agit en aucun cas, en revanche, de prétendre qu'un savoir doit forcément être lié à l'expérience directe pour être utile et efficace ; nous estimons que, s'agissant du langage, il est impératif que les formes linguistiques apprises et manipulées en 
Hamma, B. (2019a), in Dir. Cécile Avezard-Roger, Céline Corteel, Jan Goes, Belinda Lavieu-Gwozdz: La phrase, carrefour linguistique et didactique. Artois Presses Université, p. 281-301.

classe soient associées à l'usage réel de la langue : c'est un gage de discernement et d'assimilation.

\subsection{La phrase forgée dans le discours didactique: implications et pistes de changement}

\subsubsection{Le cas de la litote}

La litote apparaît le plus souvent dans une liste de figures de style ou dans un tableau que l'on donne habituellement en annexe dans les manuels ou dans les livres destinés aux concours de recrutement des professeurs des écoles ${ }^{3}$. Il est généralement laissé à la bonne volonté du formateur, qui choisira quand y avoir recours lors de l'explication d'extraits de la littérature française classique ou moderne. Ainsi, la litote est définie comme une figure d'atténuation qui permet de «Dire moins pour suggérer plus » et l'exemple le plus cité est «Va, je ne te hais point! ». Ce qui nous paraît contestable dans la présentation de cette figure, c'est à la fois la manière uniformisée et normalisée de la définir et la circularité de l'explication, puisqu'il y a très peu de manuels qui choisissent de reformuler cette définition en utilisant d'autres mots, sans parler du fait que l'exemple repris pour l'illustrer paraît à la fois anachronique et obscur ! En effet, dans la tête de plusieurs générations, il y a une cristallisation du sens de la litote, de l'exemple d'identification et de la glose. De fait, dès que l'on demande un autre exemple, ou une reformulation de la définition donnée, l'apprenant se trouve démuni et, dans le meilleur des cas, il produit des exemples en jouant sur l'axe paradigmatique à partir des exemples qu'il avait appris par cœur, or il serait assez difficile de défendre la présence d'une litote dans un exemple comme Je ne te déteste pas, calqué sur celui de Corneille.

Il aurait été plus constructif de choisir, au moins dans un premier temps, des exemples qui parlent aux élèves et non ces phrases usées que l'on perpétue et qui deviennent dénuées de tout sens. Ainsi, on oublie que des cas aussi banals que : Il est loin d'être bête ! ou aussi Il est pas mal du tout, son dernier clip! ; Il ne se laisse pas faire! ; etc. pourraient permettre d'atteindre plus rapidement et plus efficacement les objectifs du cours avec une explication claire de ce qu'est la litote. Les natifs s'en servent et en connaissent très bien les effets et peuvent les identifier aisément, vu qu'ils sont issus de la pratique commune de leur langue maternelle, en tout cas, bien plus que les exemples que 1'on cite habituellement dans les manuels. D'un autre côté, ces exemples pourront très bien être réutilisés dans un échange ordinaire par les apprenants de FLE (on retrouve ainsi une qualité certaine : la possibilité de réemploi). Voilà deux facteurs qui sont susceptibles de rendre l'acquisition de ce fait plus aisée, plus sensée et, par conséquent, plus durable. Etendre l'exemplification à la littérature écrite et soutenue par la suite ne devrait pas poser problème.

\footnotetext{
${ }^{3}$ Voir, par exemple, Lelièvre \& Vénérin-Guénez (2014) et Hiu (2016).
} 
Pour les contextes de FLE, on pourrait aussi partir d'exemples qui parlent mieux aux apprenants : pourquoi ne pas aller les chercher dans leur langue maternelle pour faciliter l'intégration conceptuelle de la notion abordée ? Avec les facilités qu'offrent le web de nos jours, cela doit se faire sans encombre et si on avait des doutes concernant la prononciation, ce serait une occasion inestimable de mettre en valeur les aptitudes des apprenants et de les impliquer davantage, aussi bien pour lire les exemples cités que pour leur demander de les gloser ou d'en donner les contextes d'emploi. Pour des anglophones, par exemple, on pourrait reprendre des énoncés comme Not bad! ; I don't unlike! ; not too shabby! Après, on pourra passer aux exemples français les plus ordinaires, du type Pas mal! ; Il est pas bête! ; C'est pas très catholique ; C'est pas une partie de plaisir ; C'est pas joli joli ! ; Etc.; ainsi, on pourra leur faire remarquer, par exemple, que ces formes tendent au figement, le ne de négation et être y sont généralement effacés. Pour aller plus loin, on pourrait évoquer une propriété orale assez cruciale, à savoir la distinction du sens de l'énoncé en fonction de la manière de le prononcer ; il existe un pas mal litotique avec un allongement de la dernière syllabe, qu'il ne faudrait pas confondre avec le pas mal, prononcé sans allonger la dernière syllabe et souvent de manière nonchalante, ce qui amène à rappeler éventuellement la mimique accompagnant ces tournures. Ils pourront alors distinguer le degré de satisfaction du locuteur : qui sera mitigé pour la dernière occurrence (signifiant «ça peut aller») ou extrême, pour le cas de la litote (signifiant alors « excellent/fantastique »).

Enfin, on pourrait, dans la phase de sensibilisation, en FLE, comme en FLM, partir d'exemples banals de la vie quotidienne, pris dans un contexte moins académique, dans un premier temps, pour aborder ensuite des contextes plus formels et plus subtils. Ainsi, pour assurer cette transition, on pourrait leur demander de trouver un équivalent ou de l'exprimer dans un langage plus formel, relevant de l'écrit ou d'un registre plus soutenu, ce qui permet d'avoir des exemples proches de ceux que l'on trouve dans la production littéraire. Et si l'on voit qu'ils ont bien saisi le sens de cette figure, on pourrait leur demander d'en fournir une définition avec leurs propres mots (dans le cadre d'une stratégie inductive par exemple); ils ne choisiront certainement pas une définition comme « Dire le moins pour suggérer le plus »! Des élèves qui auraient découvert ces faits de cette manière auraient sans aucun doute donné de meilleures réponses pour notre questionnaire.

\subsubsection{Le cas du passif}

Nos remarques sur le passif se fondent sur l'observation d'exemples pris dans différents manuels de FLE, allant du niveau A2 au niveau B1 ${ }^{4}$, de FLM (pour les niveaux CM1-CM2) et de certaines ressources en ligne qu'utilisent fréquemment les enseignants et les apprenants de français, comme lepointdufle.net (PFN), dont nous reprenons ici une sélection que nous pensons représentative du type d'outils utilisé. Les phrases actives (1-5)

\footnotetext{
${ }^{4}$ Selon le CECRL (p. 25), le niveau A2 correspond au niveau de « survie » chez un utilisateur élémentaire (débutant) et le niveau B1 correspond au niveau « seuil » chez un utilisateur indépendant (intermédiaire).
} 
Hamma, B. (2019a), in Dir. Cécile Avezard-Roger, Céline Corteel, Jan Goes, Belinda Lavieu-Gwozdz: La phrase, carrefour linguistique et didactique. Artois Presses Université, p. 281-301.

apparaissent dans le volet «entraînement» et servent de support pour faire la transformation passive :

1. La famille regarde la télévision (PFN).

2. Nathalie récita un poème (PFN).

3. On a raté le train $(P F N)$.

4. Le dentiste soignait mes dents $(P F N)^{5}$.

5. Gabriel refuse notre aide (BLED CM1/CM2, 2008)

Si l'on applique scrupuleusement la règle apprise, cela donnerait a priori les transformations suivantes, en (1a-5a). Néanmoins, si l'on juge ces phrases du point de vue de leur possibilité d'usage dans le discours et de leur intérêt discursif réel, on s'aperçoit qu'elles deviennent toutes douteuses et méritent, au moins, un point d'interrogation ou deux

1a. ??La télévision est regardée par la famille.

2a. ?Un poème fut récité par Nathalie.

3a. ?? Le train a été raté.

4a. ??Mes dents étaient soignées par le dentiste.

5a. ?Notre aide est refusée par Gabriel.

Considérons de près ce qui ne va pas dans ces transformations. Selon nous, le sentiment de bizarrerie s'explique avant tout par le fait que, contrairement à la forme active d'origine, la version passive ne paraît pas mettre en scène de vraies situations de communication. Or une phrase en cache souvent une autre. Il est, en effet, indispensable d'anticiper et de jauger aussi les implications transformationnelles dans un exercice pour éviter de noyer l'élève dans le non-sens. Ainsi, pour les phrases (1) et (2), c'est la qualité informationnelle qui est en cause dans les transformations obtenues; en fait, l'aspect «imperfectif» et "non accompli » que véhicule la forme verbale regardent, un procès en cours de réalisation, se trouve en conflit avec la notion d' «accompli » impliquée par le passif périphrastique en (1a). Cette bizarrerie s'explique aussi par l'absence de «saturation sémantique » (Cadiot, 1991, p. 109) ; c'est d'ailleurs le cas aussi de (2a) : le SN indéfini Un poème semble à son tour incomplet et insuffisant. Pour que ces phrases soient sémantiquement saturées, et partant, plus naturelles, il aurait fallu, en l'occurrence, ajouter un complément d'information consistant, par exemple, en un ajout marquant une certaine «fréquence », pour améliorer (1a), ce qui impliquerait un changement au niveau de l'enjeu communicatif : l'apport informationnel se trouverait alors au niveau du circonstant, comme en (1b), ou aussi d'une « relative » permettant de mieux identifier le sujet (2b) et, éventuellement, un complément de phrase, ou même un second prédicat qui succèderait au premier dans une

\footnotetext{
${ }^{5}$ Notons que la version active de (4) pris du PFN serait plus naturelle ainsi : Le dentiste me soignait les dents.
} 11 
Hamma, B. (2019a), in Dir. Cécile Avezard-Roger, Céline Corteel, Jan Goes, Belinda Lavieu-Gwozdz: La phrase, carrefour linguistique et didactique. Artois Presses Université, p. 281-301.

liste d'actions (2c) pour permettre une meilleure saturation de l'information que véhicule l'exemple (2a) :

\section{1b. La télévision est regardée par la famille seulement deux fois par semaine}

2b. Un poème (que lui avait recommandé Marie + qui lui a été recommandé par Marie) fut récité par Nathalie devant toute la classe ;

2c. Un poème fut récité par Nathalie (puis mimé + puis distribué à toute la classe).

Avec ces quelques insertions, le contenu informationnel se trouve ainsi saturé et la bizarrerie semble s'estomper en comparaison avec les deux exemples de départ. On pourrait se demander, à ce stade, pourquoi les acteurs pédagogiques ne s'aperçoivent pas forcément de ces anomalies qualitatives, si évidentes. La réponse est loin d'être simple : d'une part, le formateur, par souci de clarté, peut se trouver contraint de prendre des exemples concis et canoniques, ne s'écartant pas du schéma habituel et, idéalement, sans " aspérités », en vue de montrer simplement de quelle manière la règle opère. En l'occurrence, il s'agit d'expliquer les mécanismes sous-jacents à la transformation passive. D'autre part, ce type de pratiques n'est qu'un réflexe qui s'est ancré dans les habitudes scolaires depuis plusieurs décennies, un héritage des MAO et SGAV depuis les années 50-60. Ces approches présument que la langue est un réseau de structures et un ensemble d'automatismes qu'il faudrait apprendre d'une manière irréfléchie et machinale. Cette vision du monde repose, en fait, sur les postulats structuralistes innéistes et behaviouristes qui insistent sur le côté inconscient et incontrôlé du processus d'acquisition linguistique et prônent de fait la forme aux dépens du sens et de l'usage : les exemples dans ces perspectives n'ont pour but que de renforcer un comportement verbal, une structure abstraite dans l'esprit. Mais, le prix à payer justement, c'est que les phrases prises et les transformations qui en découlent peuvent paraître très bizarres et très éloignées de la réalité des pratiques langagières. On pourrait, à première vue, concéder à ce type d'approche la commodité de la procédure, étant donné la pertinence incontestable d'une démarche utilisant des exemples simples et concis, dans une optique constructiviste, mais ce qui est critiquable, dans ces méthodes, c'est quand l'exemple forgé et simplifié demeure le seul recours.

Il en va de même de l'exemple (3), où l'on relève une inadéquation entre la nature du procès et le sujet de la phrase passive obtenue. Ce type de phrase est fait, selon nous, pour rester à la forme active pour ne pas entraîner une interprétation erronée de la phrase : on penserait, par exemple, à « une maquette de train qui a été ratée », ou « qui contient de gros défauts », autrement, la phrase serait tout simplement absurde et n'aurait aucune chance d'exister, même avec une personnification du N train, ou aussi dans le scénario de certains dessins animés ; on verrait mal, par exemple, Bob le train (un personnage de dessin animé) dire :??On m'a raté ! Il n'y aurait pas, en effet, d'enjeux argumentatifs et la situation semble absente dans la mémoire discursive, du type ??Le train a été raté! Tant pis, il 
prendra les passagers suivants! Et justement, l'avantage du recours aux données authentiques est de ne jamais risquer de tomber sur ce type de phrases.

Avec la phrase (4), on assiste à trois problèmes : le premier est lié au sujet de la phrase, qui ne paraît pas naturel ; l'explication donnée par Dubois (1967) paraît à cet égard très satisfaisante dans la mesure où la langue française semble favoriser plutôt des sujets « animés » en position sujet ; on aura, par exemple, Le chasseur a capturé un lapin, mais difficilement ??Un lapin a été capturé par le chasseur, l'hypothèse de Dubois peut cependant être affinée et nuancée ( $c f$. Hamma, 2017 à par.). Quant au deuxième problème, il concerne le complément d'agent utilisé; en effet, nous avons démontré (Hamma, 2015a) que le complément en par ne peut pas renvoyer à un agent prévisible ou évident. En d'autres termes, l'énoncé tel quel paraît transgresser les lois de discours de qualité et de quantité : il est communément admis que quand une information est évidente ou connue, il est inutile de la préciser, puisqu'il est bien normal, par exemple, que l'on se fasse soigner les dents par un dentiste ou que l'on se fasse livrer par un livreur... ; c'est un fait bien ancré dans la mémoire discursive. Dans ces cas, soit on aura une occultation de l'agent (Le Goffic, 1970), soit il faudrait que le propos de l'énoncé contienne une information « marginale » ou «non évidente » sur l'agent (Hamma, 2015a), du type (4b). Ceci dit, il n'est pas complètement exclu d'avoir un agent comme par le dentiste, ici, mais avec un scénario complètement différent et sous certaines conditions. En fait, cela confèrerait à l'agent une valeur informationnelle importante et déclenche un calcul prédicatif particulier : on décrirait une certaine rupture par rapport à une habitude perçue comme plus ou moins atypique ou non conformiste : le locuteur se faisaient, par exemple, soigner les dents par quelqu'un qui s'y connaît (sa grand-mère, un barbier, etc.) et pour une fois il va voir un dentiste pour changer, mais, là aussi, on ne pourrait pas faire l'économie d'une justification co(n)textuelle très claire de la précision inopinée de l'agent, à travers différents types d'affixes, du type (4b) ou (4c), ou un appendice, comme en (4d). De même, avec un « entassement » appositif (un vrai dentiste, un vrai de vrai) en (4c) - une autre spécificité de la linguistique de l'oral que l'élève doit connaître et savoir interpréter - on aurait eu un exemple plus naturel, en tout cas, pour cette partie de la phrase (d'où le «? »restant), vu qu'on aura toujours une autre bizarrerie entraînée par l'utilisation du temps imparfait (soignait/étaient soignées), qui laisse entendre, ici, qu'il s'agit d'une habitude dans le passé et empêche, ipso facto, de prendre l'agent mentionné (par le dentiste) pour un écart à une habitude connue comme expliqué supra. Et de toute façon, avec un verbe trivalent, ici, le factitif est beaucoup plus naturel qu'un périphrastique (4e) :

4. ?Le dentiste soignait mes dents.

4a. ?Mes dents étaient soignées (par le barbier + par un ami + par ma grandmère).

4b. ?Pour une fois, mes dents ont été soignées par le dentiste! 
Hamma, B. (2019a), in Dir. Cécile Avezard-Roger, Céline Corteel, Jan Goes, Belinda Lavieu-Gwozdz: La phrase, carrefour linguistique et didactique. Artois Presses Université, p. 281-301.

4c. ?Cette fois-ci, mes dents ont été soignées par le dentiste, un vrai dentiste, un vrai de vrai!

4d. Mes dents étaient soignées par un dentiste... ma grand-mère était en voyage!

4e. Pour une fois, je me suis fait soigner les dents par le dentiste, par un vrai dentiste!

Il en va de même pour la phrase (5a), qui ne paraît pas non plus très naturelle ; outre les remarques faites précédemment pour (4) à propos de l'ordre de prédilection de ces arguments en français tel qu'imposé par la nature du sujet («inanimé ») et le poids fonctionnel, on voit aussi qu'on a du mal à y trouver un emploi pertinent où il serait dit tel quel : cet exemple sera sans doute meilleur si on avait inséré des commentaires énonciatifs, comme en (5b), pour marquer, par exemple, une résistance attendue de la part de Gabriel (il y a eu des propositions d'aide antérieurement qu'il avait repoussées) ; avec par Gabriel, on présuppose que d'autres que lui aient accepté cette aide en vertu de l'apport de contraste que véhicule un complément d'agent en par ( $c f$. Hamma, 2015a); de fait, en mentionnant explicitement cette information, l'exemple passera beaucoup mieux, comme en $(5 \mathrm{c})$, à travers les différentes insertions en gras. Ainsi, on aura un noyau impliquant "Gabriel refuse de se faire aider » et différents satellites venant légitimer cet usage et qui deviennent, de ce point de vue, indispensables pour que l'énoncé passe le test de la dicibilité.

5b. (Rien à faire + Quoi qu'on fasse) notre aide est refusée par Gabriel!

5c. Rien à faire notre aide est toujours refusée par Gabriel... les autres ont tous accepté de se faire aider, mais Gabriel, il est trop fier!

Pour les mêmes raisons que (4) le factitif serait meilleur (5d), surtout qu'il permet de mettre en position de sujet un $\mathrm{N}$ animé-humain, qui plus est connu.

5d. Rien à faire! Gabriel refuse de se faire aider.

Les phrases suivantes, telles quelles, aboutissent globalement aux mêmes types d'anomalies. Il est demandé aux élèves de les mettre à la forme active en tenant compte de ce qu'ils ont vu précédemment dans la leçon (MGCM1/CM2, 2010) :

6. Les falaises ont été sculptées par les vagues.

7. Des paniers auront été tressés par des bohémiens.

8. Mes parents ont été reçus par leurs amis.

Encore une fois, bien que syntaxiquement bien formées (6a, 7a et 8a), les transformations obtenues laissent à désirer sur le plan de leur acceptabilité et de leur interprétation :

6a. ??Les vagues ont sculpté les falaises.

7a. ??Des bohémiens auront tressé des paniers. 


\section{8a. ??Leurs amis ont été reçus par mes parents.}

En (6a), par exemple, on retrouve un problème d'aspect; le procès y renvoie davantage à la description d'un état ou, au moins, au résultat d'une action plutôt qu'à une vraie action à la forme passive, comme le montre la possibilité de le remplacer, tout en restant fidèle au sens, par un segment sans être (6b). Cette commutation entraîne, de fait, une annulation de l'ancrage dans le temps: on a un SN désignant une certaine entité avec des propriétés particulières et l'effacement du verbe y crée un effet d'attente obligeant d'ajouter un SV différent pour que l'exemple soit acceptable :

\section{6b. Les falaises sculptées par les vagues fascinent les touristes.}

En (7a), on a un sujet inconnu, mal identifié : ici, la bizarrerie constatée concerne aussi bien le sujet actif (Des bohémiens) que le sujet passif (Des paniers). De fait, un emploi endophorique ou au moins indexical (nos/ces paniers), par exemple, aurait permis d'améliorer l'exemple. De plus, le procès de la phrase est au futur antérieur (auront tressé/auront été tressés). Ce temps relatif, marquant ordinairement une certaine antériorité par rapport à une référence événementielle donnée, paraît complètement injustifié sans contexte : cela provoque encore une fois un problème de saturation.

Quant à la transformation en (8), elle contient un problème de référence qu'entraîne le passage de la forme passive à la forme active ; en effet, le possessif leurs devient ambigu dans la version active obtenue, puisqu'il implique un changement au niveau de celui qui invite; dans la forme passive, il s'agit a priori des amis des parents alors que dans la forme active, cela peut renvoyer à un référent anaphorique, absent dans la phrase telle quelle, et qui, en tout cas, n'est pas coréférentiel de l'agent passif de la phrase citée.

Ainsi, ces différents exemples réduisent le tour passif à une simple manipulation scolaire, que l'on ne rencontre que dans un cours de français, une tournure rigide dont l'intérêt discursif n'est guère évoqué. Nous ne prétendons pas que les phrases doivent être obligatoirement contextualisées pour qu'elles soient naturelles et qu'elles aient un sens ; on peut, tout à fait, avoir des exemples qui sont à la fois simples et cohérents $(1 \mathrm{~b} ; 2 \mathrm{~b}-2 \mathrm{c} ; 4 \mathrm{e}$; $5 \mathrm{~d} . .$.$) . On pourrait, par exemple, réfléchir aux situations appelant l'emploi du passif,$ comme le font déjà certains manuels $(c f . G P F)$, où l'on associe ce tour aux inventions ou aussi aux faits divers, ce qui permet déjà de s'éloigner des drills traditionnels. Mais ces quelques stratégies restent isolées dans ces manuels, vu que dans le reste de la leçon, on retrouve les mêmes généralisations classiques. Selon nous, un travail sur les notions de « contraste » et de « non-conformisme » impliquées par la précision de l'agent ou aussi sur les considérations aspectuelles affectant le procès peut fournir un meilleur ancrage dans l'usage. 
Notons, par ailleurs, que le recours aux phrases forgées conduit souvent à sacrifier les emplois passifs non-canoniques qui représentent pourtant plus de la moitié des passifs dans l'usage, d'après les ESLO ( $c f$. figure ci-dessous), soit $43 \%$ contre $57 \%$, sur une base de 3598 énoncés. C'est généralement l'emploi périphrastique qui est privilégié dans les manuels de FLE et de FLM examinés, à quelques exceptions près, où l'on mentionne timidement les emplois pronominal et factitif. Or, dans la catégorie des emplois «noncanoniques $"$, les passifs pronominaux représentent seulement $8 \%$ et les factitifs-tolératifs $1 \%$, face aux adjectifs déverbaux en -ble à sens passif (34\%) ou aussi les GN passifs, avec une enchâssée relative ou avec un effacement du verbe être $(13,7 \%)$ et les tournures en $A D J$ $+\grave{a}+\operatorname{Vinf}(5 \%)$. Donc, on voit bien que l'intérêt accordé à certaines formes aux dépens d'autres ne se justifie pas du point de vue de l'usage, comme le montrent les chiffres dans les ESLO.

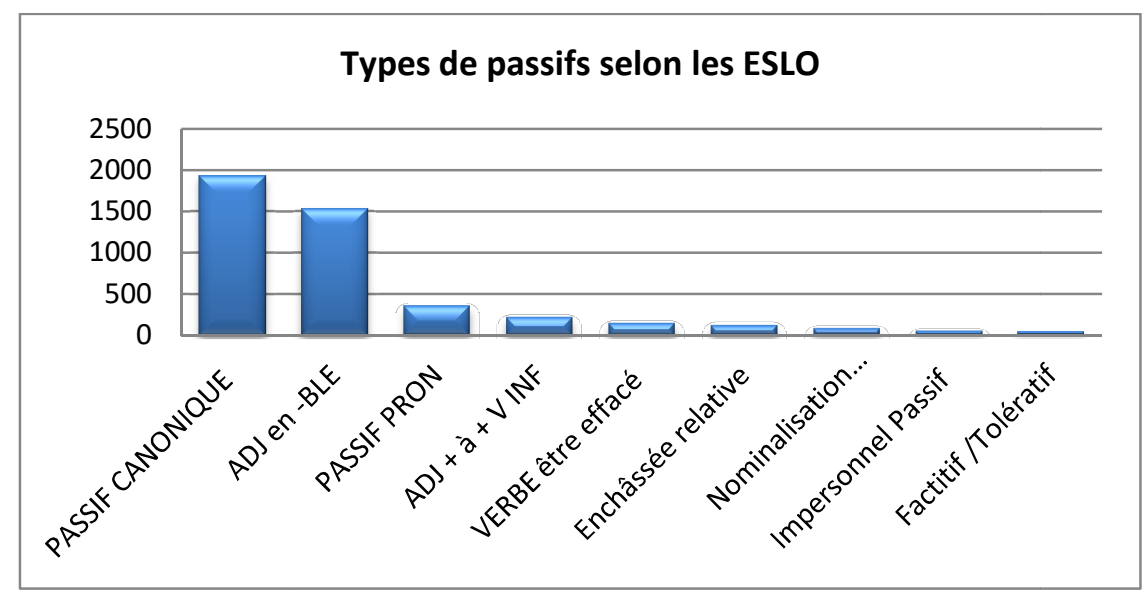

Répartition des emplois passifs dans les ESLO (Hamma, 2014a)

Ainsi, beaucoup d'emplois se trouvent paradoxalement marginalisés au profit d'un canon qui n'est, tout compte fait, pas représentatif des emplois passifs du français. Notons d'ailleurs que les phrases forgées périphrastiques sont généralement formées d'une manière identique : on a toujours un nom propre ou commun en guise de sujet. Or, notre corpus ESLO montre que le sujet passif est un pronom anaphorique dans plus de $95 \%$ des occurrences passives (Hamma, 2014a) : cela anaphorise le plus souvent un nom connu, déjà évoqué dans le contexte ou renvoie à un indexical. Les rares noms communs relevés renvoient à un référent générique ou évoqué antérieurement. Si l'utilisation des noms propres paraît en revanche possible, c'est parce qu'ils sont autosuffisants du point de vue référentiel (ils représentent leur référent de manière univoque); or ce qui est souvent remarqué dans les manuels examinés, justement, c'est le fait d'utiliser des noms propres. Ainsi, Le roman Fermina Márquez a été écrit par Valery Larbaud (GR \& Exp 4e 1998) ou Notre équipe de football est dirigée par M. Lévêque (NGF, 2004) sont trompeurs : le nom propre permet, en réalité, de dispenser du contexte, mais sans que l'élève comprenne pourquoi. Et de toute façon, ces deux exemples, tels quels, semblent, très curieux à la voix 
active et nécessitent quelques ajustements: ?? Valery Larbaud a écrit le roman Fermina Márquez et ?M. Lévêque a dirigé Notre équipe de football. De plus, comme les exemples cités sont le plus souvent forgés ou décontextualisés, on s'aperçoit que certaines situations sont complètement absentes des manuels usuels de français. En effet, un «segment passif» n'est pas toujours l'unité phrastique supérieure dans le discours ; il peut occuper, du point de vue macrosyntaxique, aussi bien le statut de noyau énonciatif, d'incise, que différents types d'affixes, selon la terminologie proposée par Blanche-Benveniste (1984). Ainsi, dans l'énoncé (9), le segment passif en gras occupe une position d' «incise »; son rôle « parenthétique » et « anticipatif » est au cœur de l'explication de ce tour : il s'agit, ici, d'un cas de prédication seconde consistant à apporter une précision que le locuteur juge importante et qui ne paraît pas aller de soi (Hamma, 2015a) :

9. ...mais mon fils leur a dit bah ça va fermer ça parce que vous allez devoir retravailler et n'oubliez pas bande d'idiots - il est il les a il est bien aimé par ses ouvriers - il dit si vous cassez tout et puis qu'est-ce que comment vous travaillerez demain? (ESLO 1).

De même, dans (10-12), le segment passif occupe plutôt une position affixale : il est préfixe ou topique en (10), suffixe en (11) et postfixe en (12); l'information véhiculée par ces segments reste dans ces différents cas de figure importante (c'est une prédication seconde) :

10.... ce qui est contestable c'est la manière dont l'autorité est exercée

11... et finalement l'ensemble du pays était mécontent la meilleure preuve c'est que la machine s'est arrêtée pendant trois semaines bloquée par les grèves

12.... y a eu X qui a été ministre il l'a pas été longtemps il a été tué par sa femme

$\mathrm{Du}$ point de vue rectionnel (donc microsyntaxique), le segment passif rentre aussi dans différents types de relations avec les segments avoisinants qu'il faudrait impérativement prendre en compte si l'on veut que l'explication didactique soit efficace et non partielle. On voit par exemple qu'il est complément de $\mathrm{N}$ dans (13); il est enchâssé dans un SN et est, d'ailleurs, non supprimable a priori pour des raisons de saturation sémantique :

13. ...parce que je trouve dommage que des personnes qui sont entendues par des milliers de de Français euh parlent très mal

Au vu de ces quelques observations, on pourrait dire que l'enseignement du passif à l'heure actuelle ne rend pas justice à l'ensemble des emplois observables de ce tour dans le discours et mérite, de fait, d'être repensé et complété par une approche où l'oralité trouvera sa place aux côtés de l'écrit. Et, à moins de soutenir qu'il existe un genre de discours propre à l'école et qui aurait pour particularité d'être complètement détaché de la réalité des pratiques langagières - ce qui serait absurde et déroutant -, les phrases forgées doivent être écartées ou au moins doivent être établies avec plus de soin. Ce qui est préjudiciable dans le 
recours aux phrases forgées dans l'exemplification scolaire, c'est le fait que l'élève manipule des formes qui n'ont pas réellement de consistance discursive et, du point de vue de la praxis, les savoirs appris ne sont réutilisables a priori que le jour de l'examen, ce qui ne garantit pas le fait qu'il soit en mesure de les identifier, ou de les produire sous une autre forme ultérieurement, comme le montrent les résultats de notre questionnaire.

\subsection{Bilan, perspectives et conclusions}

La solution aux problèmes relevés n'est pas tant dans le fait de contrebalancer la prédilection pour l'écrit en ouvrant les portes de l'école à l'oral, que d'œuvrer à accoutumer les élèves à la distinction entre ces deux genres de discours, qui n'ont pas les mêmes outils de description, ni le même domaine d'application. Une telle entreprise suppose une prise en compte des énoncés oraux issus des interactions verbales spontanées et une extension de l'unité phrastique à l'unité dialogique, de manière à compléter le recours quasi exclusif dans l'exemplification scolaire à l'écrit et aux textes d'auteurs, et partant, à supplanter les phrases forgées et décontextualisées par des énoncés «vivants ", ce qui fournirait à l'esprit de l'apprenant un meilleur produit pour l'acquisition et pour le développement des compétences communicatives et socio-pragmatiques. De fait, aussi bizarre que cela puisse paraître, la solution que l'on peut proposer en vue d'améliorer, d'un côté, les compétences orales en FLE et, de l'autre, les compétences écrites en FLM est d'œuvrer à introduire et à renforcer la compétence diamésique en classe de français, en familiarisant les apprenants avec les spécificités du discours oral. L'intérêt de l'oral pour le FLM se voit aussi dans une meilleure assimilation des faits linguistiques étudiés. Une telle entreprise implique une utilisation lucide des corpus oraux et des outils de description appropriés.

Afin de mettre en pratique ce type de compétences, on pourrait élargir les objectifs pédagogiques en proposant aux élèves des activités de transposition les invitant, par exemple, à passer de l'écrit à l'oral, et inversement ; ainsi, ils pourraient prendre conscience du fait que l'énoncé oral renferme différents phénomènes linguistiques qui lui sont propres et qui, d'ailleurs, peuvent être glosés et exploités dans la compréhension ou l'explication des dialogues. Ce type de tâche pourrait se décliner en différents objectifs spécifiques, comme l'expression du possessif à l'oral par opposition à l'écrit (Ma voiture vs Ma voiture à moi/ Moi, ma voiture, etc.), ou aussi l'éventail des temps verbaux utilisés dans chaque genre de discours ( $c f$. futur périphrastique vs futur simple...) ; les pronoms ( $c f$. on vs nous ; $i l$ vs $i$ vs $\varnothing \ldots$.$) et d'autres phénomènes, comme les détachements, les reprises, les$ répétitions, surtout pour l'oral et l'utilisation des connecteurs, surtout pour l'écrit ; etc.). L'une des consignes possibles pourrait être aussi de demander à l'apprenant d'éviter les répétitions ou de proposer une explication ou une suite aux amorces contenus dans une prise de parole spontanée ; etc. Et pour veiller à la représentativité des énoncés, on pourrait jouer sur certaines variables, entre autres, en imaginant d'autres situations de communication mettant en scène des interlocuteurs ayant des profils variés (des hommes, des femmes, des jeunes, des personnes âgées, des enfants, des locuteurs issus de divers 
milieux socioprofessionnels et de régions différentes), ce qui est susceptible d'entraîner différents types de variations sociolinguistiques, aussi bien au niveau lexical que grammatical.

Ainsi, le recours à l'oral est en mesure de permettre, à la fois, de compléter les supports pris dans des textes d'auteurs où l'écrit est en vedette et de supplanter le caractère artificiel des données forgées rencontrées dans certains manuels. Cette prise en compte des deux types de données a l'avantage, sur le plan cognitif, de familiariser les apprenants avec ces subtilités, en enclenchant chez eux un processus de prise de conscience des aspects diamésiques caractérisant le code du français dans ses diverses actualisations.

\section{Références bibliographiques}

BLANCHE-BENVENISTE C., (1984), Pronom et Syntaxe. L'approche pronominale et son application au français, Paris, Selaf-Aelia.

BOURDIEU, P. (1982), Ce que parler veut dire: L'économie des échanges linguistiques Paris. Éditions Fayard.

CADIOT, P. \& NAOYO, F (2000), « Présentation ». In : Langue française, n $125: 3-5$.

CADIOT, P. (1991), De la grammaire à la cognition: la préposition pour. Éditions du Centre National de la Recherche Scientifique.

CORDIE, A. (1993). Les Cancres n'existent pas. Psychanalyses d'enfants en échec scolaire. Editions du Seuil.

DUBOIS, J. (1967), Grammaire structurale du français. Le verbe. Paris, Larousse.

GADET, F. (1996), "Une distinction bien fragile: oral/écrit». Travaux neuchâtelois de linguistique, $\mathrm{n}^{\circ} 25, \mathrm{p} .13-27$.

GOFFMAN, E. (1973), La Mise en scène de la vie quotidienne, t. 1 La Présentation de soi ; t. 2 Les Relations en public. Éditions de Minuit, coll. «Le Sens Commun ».

GUERIN, E. (2014), « Le "français langue maternelle" est-il une "langue vivante" ? Réflexion sur la place de la variation stylistique dans le discours scolaire », in Lidil, 50 Variation stylistique et diversité des contextes de socialisation. En jeux sociolinguistiques et didactiques : 147-167.

HAMMA, B. (2015a), "Agent passif en par et sujet actif: les dessous d'un contraste », Revue de Sémantique et Pragmatique. Numéro 37 : 61-83.

HAMMA, B (2017, à par.), «Quand l'interaction n'est pas là, la souris est mangée par le chat! Remarques sur l'enseignement du passif en classe de français ». Actes de la Journée d'études Interaction(s) et didactique des langues. PUFC.

HAMMA, B. (2014a), Emplois non-canoniques du passif à l'oral. Journées FLORAL, 4 décembre 2014, Cité Internationale Universitaire, Maison de Norvège.

HAMMA, B. (2014b), Le passif, étude sur corpus et applications didactiques. Journées FLORAL, 8 décembre 2014, Cité Internationale Universitaire, Maison de Norvège. 
Hamma, B. (2019a), in Dir. Cécile Avezard-Roger, Céline Corteel, Jan Goes, Belinda Lavieu-Gwozdz: La phrase, carrefour linguistique et didactique. Artois Presses Université, p. 281-301.

LE BELLEC, C. \& HAMMA, B. (2014), « Le traitement du passif dans l'enseignement du français langue étrangère ", in Lidil, 50 Variation stylistique et diversité des contextes de socialisation. En jeux sociolinguistiques et didactiques : 191-211.

LE GOFFIC, P. (1970), «Linguistique et enseignement des langues : à propos du passif en français ». In Langue française 8. Apprentissage du français langue étrangère. Paris : Larousse : 78-89

MIONI, A. (1983), « Italiano tendenziale : Osservazioni su alcnia spetti della standardizzazione », in Scritti linguistici in onore di Giovan Battista Pellegrini, a cura di P. Benincà et al., Pisa, Pacini, 2 voll., vol. $1^{\circ}$, pp. 495-517.

RAVON, B. (2000), L'échec scolaire. L'histoire d'un problème public. Paris, In press.

TISSET, C. (2010), Enseigner la langue française à l'école, Coll. Profession Enseignant. Hachette éditions.

VIGNER, G. (2003), " Nommer le français ». Revue de didactologie des langues-cultures, $130: 153-166$.

WÜEST, J. (2009) «La notion de diamésie est-elle nécessaire ?. », Travaux de linguistique $2 \mathrm{n}^{\circ} 59: 147-162$

\section{Corpus :}

ESLO (Enquêtes SocioLinguistiques à Orléans): $\underline{\text { http://eslo.huma-num.fr/ }}$

\section{Manuels scolaires et préparation au CNPE}

ACHARD, A.-M., et al. (1998), Grammaire et expression 4e. Hachette Éducation (Gr \& Exp $\left.4^{e}\right)$.

BARRAGE, F. \& MÜNZER, A. (2010), Manuel de grammaire CM1-CM2. La librairie des écoles $(M G)$.

DELATOUR, Y., et al. (2004), Nouvelle grammaire du français : Cours de Civilisation Française de la Sorbonne Relié. Paris. Hachette (NGF).

GRÉGOIRE, M. \& THIEVENAZ, O. (2002), Grammaire progressive du français. Avec 600 exercices. CLE International. $(G P F)$

HIU, J. (2016), Annales CRPE 2017 : Français. Nathan. Paris

LELIEVRE, S. \& VENERIN-GUENEZ, C. (2014), Préparer et réussir le CRPE. SEDES. Paris.

\section{Référentiels de formations}

BO (2015) : Le Bulletin officiel de l'éducation nationale : http://.education.gouv.fr

CERCL (2011) : Cadre Européen Commun De Référence pour les Langues: Apprendre, Enseigner, Evaluer : www.coe.int/lang-CECR

SCCCC (2016): Socle Commun de Connaissances, de Compétences et de Culture: $\underline{\mathrm{http}: / / \text { eduscol.education.fr }}$ 